\title{
Corrigendum
}

O'Brochta, D. A., Sethuraman, N., Wilson, R., Hice, R. H., Pinkerton, A. C., Levesque, C. S., Bideshi, D. K., Jasinskiene, N., Coates, C. J., James, A. A., Lehane, M. J. and Atkinson, P. W. (2003). Gene vector and transposable element behavior in mosquitoes. J. Exp. Biol. 206, 3823-3834.

A paper by Adelman et al. was incorrectly cited in the text and Reference list as 'in press' in Genetics. The article has actually been submitted elsewhere.

The authors apologise for any inconvenience this may have caused. 


\title{
Review
}

\section{Gene vector and transposable element behavior in mosquitoes}

\author{
David A. O’Brochta ${ }^{1, *}$, Nagaraja Sethuraman ${ }^{1}$, Raymond Wilson ${ }^{2, \dagger}$, Robert H. Hice ${ }^{3}$, \\ Alexandra C. Pinkerton ${ }^{3, \ddagger}$, Cynthia S. Levesque ${ }^{3}$, Dennis K. Bideshi ${ }^{3}$, Nijole Jasinskiene ${ }^{4}$, \\ Craig J. Coates ${ }^{5}$, Anthony A. James ${ }^{4}$, Michael J. Lehane ${ }^{2}$ and Peter W. Atkinson ${ }^{3}$ \\ ${ }^{1}$ Center for Biosystems Research, University of Maryland Biotechnology Institute, College Park, MD 20742-4450, \\ USA, ${ }^{2}$ School of Biological Sciences, University of Wales, Bangor, Gwynedd LL57 2UW, UK, ${ }^{3}$ Department of \\ Entomology, University of California, Riverside, CA 92521, USA, ${ }^{4}$ Department of Molecular Biology and \\ Biochemistry, University of California, Irvine, CA 92697, USA and ${ }^{5}$ Department of Entomology, Texas A\&M \\ University, College Station, TX 77843-2475, USA \\ *Author for correspondence (obrochta@umbi.umd.edu) \\ $\dagger$ Present address: Division of Biochemistry, School of Biological Sciences, University of Manchester, Oxford Road, Manchester M13 9PT, UK \\ ‡Present address: Lion Bioscience Inc., 9880 Campus Point Drive, San Diego, CA 92121, USA
}

Accepted 18 July 2003

\begin{abstract}
Summary
The development of efficient germ-line transformation technologies for mosquitoes has increased the ability of entomologists to find, isolate and analyze genes. The utility of the currently available systems will be determined by a number of factors including the behavior of the gene vectors during the initial integration event and their behavior after chromosomal integration. Post-integration behavior will determine whether the transposable elements being employed currently as primary gene vectors will be useful as gene-tagging and enhancertrapping agents. The post-integration behavior of existing insect vectors has not been extensively examined. Mos 1 is useful as a primary germ-line transformation vector in

A. aegypti, but its mode of integration in mosquitoes results in the insertion of flanking plasmid DNA. Hermes can be remobilized in the soma of $A$. aegypti and transposes using a common cut-and-paste mechanism; however, the element does not remobilize in the germ line. piggyBac can be used to create transgenic mosquitoes and occasionally integrates using a mechanism other than a simple cut-and-paste mechanism. Preliminary data suggest that remobilization is infrequent. Minos also functions in mosquitoes and, like the other gene vectors, appears to remobilize inefficiently following integration. These results have implications for future gene vector development efforts and applications.
\end{abstract} insects but is inefficiently remobilized in Drosophila melanogaster and Aedes aegypti. Hermes transforms $D$. melanogaster efficiently and can be remobilized in this species. This element is also useful for creating transgenic
Key words: mosquito, transgenic insect, transposable element, Hermes, mariner, piggyBac, Minos.

\section{Introduction}

Creating the technology to produce transgenic insects has been a goal of insect molecular geneticists for years because of the great need to have effective tools to find, isolate and analyze insect genes and to genetically modify insects for the purposes of insect control. Currently, there are at least four gene-vector systems derived from Class II transposable elements that can be employed to generate transgenic nondrosophilid insects [Hermes, Mosl (mariner), Minos and piggyBac]. (Class II transposable elements move by the element precisely excising itself from its current location and reinserting itself in a new chromosomal location. This mechanism of movement is referred to as a cut-and-paste movement and is conservative in that it does not involve the replication of the element.) These systems are proving useful but their behavior can be unpredictable in the sense that, short of directly testing its mobility in the germ line, there are no good indicators of the potential effectiveness of a specific element in a species. While Hermes, Mos1, Minos and piggyBac have excellent host ranges, it is still impossible to predict their behavior in untested species. Elements may function efficiently in one species but inefficiently or not at all in close relatives. Hermes, for example, efficiently $(>50 \%)$ transforms Drosophila melanogaster but transforms Aedes aegypti with frequencies of less than $10 \%$ (Jasinskiene et al., 1998; O'Brochta et al., 1996). piggyBac can efficiently transform Anopheles albimanus but appears to be much less 
inefficient in Anopheles gambiae (Grossman et al., 2001; Perera et al., 2002). mariner (Mos1) moves efficiently in Drosophila mauritiana yet is almost immobile in $D$. melanogaster (Bryan et al., 1987; Lidholm et al., 1993). Himarl from Haematobia irritans moves efficiently in bacteria but has yet to show any evidence for mobility in insects (Lampe et al., 2000). In none of these cases was the mobility properties of the element predicted prior to use. Clearly, our understanding of these elements remains incomplete, and one aspect that remains largely unexplored is the behavior of these elements after they have become integrated into a foreign genome.

The post-integration behavior of insect gene vectors is particularly relevant to a number of future applications of this technology. Stability of integrated vectors is of concern to some researchers because genotypes created using these vectors may need to be maintained without change over time and would be of limited use in the laboratory or field if they were changing position within a genome or being lost from it. To those responsible for assessing the risks associated with releasing transgenic insects into the environment the issue of stability has additional significance. Instability, particularly if it is unpredictable, increases the uncertainty associated with describing accurately the phenotype of the insect and any attempt to model the behavior of a transgene over time in the genetically modified species.

The use of gene vectors for the purposes of creating insects with novel genotypes and phenotypes is only one of their many uses. Under some conditions, the transposable element-based gene vectors can be used to find and isolate genes by a variety of methods such as enhancer trapping and transposon mutagenesis, all of which rely on post-integration mobility or remobilization of the element. (Remobilization will be used to refer to the transposition of an insect gene vector after it has become integrated into a host's genome.) Futuristic plans for manipulating the genotypes and phenotypes of wild populations of insects such that they are no longer considered 'pest' species requires the introduction and rapid transmission of transgenes through a population. Successful implementation of such a widespread genetic transformation might be accomplished by linking the transgene to an active transposable element and taking advantage of the ability of the element to spread or drive through populations under certain conditions. Hence, assessing the potential of existing insect gene vectors to serve as genetic drives requires that the post-integration behavior of the elements be thoroughly investigated. Here, we review and report on the post-integration behavior of the Hermes, Mos1, piggyBac and Minos elements in mosquitoes.

\section{Hermes}

\section{Transposition}

The Hermes element has been shown to transpose into the germ lines of several insect species by a cut-and-paste (conservative) mechanism of transposition (Michel et al., 2001; O'Brochta et al., 1996). In these insects, only those sequences on the vector-containing plasmid that are flanked by the terminal inverted repeats of the Hermes elements were integrated into the insect genome. Furthermore, target-site duplications $8 \mathrm{bp}$ long were created at the site of insertion that conformed to the consensus sequence of $5^{\prime}$-GTnnnnAC-3' observed for other $h A T$ elements. Canonical cut-and-paste type transposition events, however, have never been observed in the germ lines of mosquitoes transformed using Hermes vectors. For both A. aegypti and Culex quinquefasciatus, germ-line integrations have involved not only all of those sequences delimited by the terminal inverted repeats of Hermes but also sequences flanking the element on the vector-containing plasmid (Allen et al., 2001; Jasinskiene et al., 1998, 2000; Kokoza et al., 2000; Moreira et al., 2000; Pinkerton et al., 2000).

Two forms of the Hermes element have been used in germline transformation experiments in mosquitoes, and both exhibit non-canonical integration patterns. The two types of Hermes elements that have been employed differ only in the terminal nucleotide of the right-hand inverted terminal repeat (ITR) sequence (Warren et al., 1994). These alternate forms of the right ITR, referred to as B5 and B6, involve a G-to-C transversion in the terminal nucleotide and originated during the isolation of two independent genomic DNA fragments from Musca domestica containing a Hermes element (Warren et al., 1994; Fig. 1). Elements containing the $B 5$ end (with a terminal $\mathrm{G})$ have been used in most of the studies of Hermes in insects (Allen et al., 2001; Michel et al., 2001; Pinkerton et al., 1996, 2000; Sarkar et al., 1997a,b), while the element containing the B6 ITR (with a terminal C) has been used in A. aegypti transformation experiments reported by others (Jasinskiene et al., 1998; Kokoza et al., 2000; Moreira et al., 2000).

We have examined the functionality of the Hermes B6 ITR in $D$. melanogaster to determine if it could also transpose by a cut-and-paste mechanism. A donor plasmid containing the B6 end and flanking $M$. domestica genomic sequence (Fig. 1) was constructed and is identical in every other way to the donor plasmid employed in previous studies. Hermes element plasmid-to-plasmid transposition assays were performed in developing $D$. melanogaster embryos using established procedures (O'Brochta et al., 1996). Transient plasmid-toplasmid transposition assays in embryos report the activity of a transposable element in somatic nuclei, since these vastly outnumber the germ-line nuclei in these embryos. Transpositions recovered from these assays are therefore most likely to originate from events occurring in somatic nuclei. We also repeated identical transposition assays using the B5 Hermes ITR, and, as previously shown, this Hermes element transposed by a cut-and-paste mechanism in Drosophila embryos (Table 1). By contrast, even though 10 times as many Hermes B6-containing donor plasmids were screened, no transposition events were recovered (Table 1). These experiments were repeated in developing A. aegypti embryos, and while Hermes $B 5$ transposition events were recovered with a frequency of $0.4 \times 10^{-2}$ per donor plasmid screened, no transpositions were recovered when the $B 6$ form of Hermes 

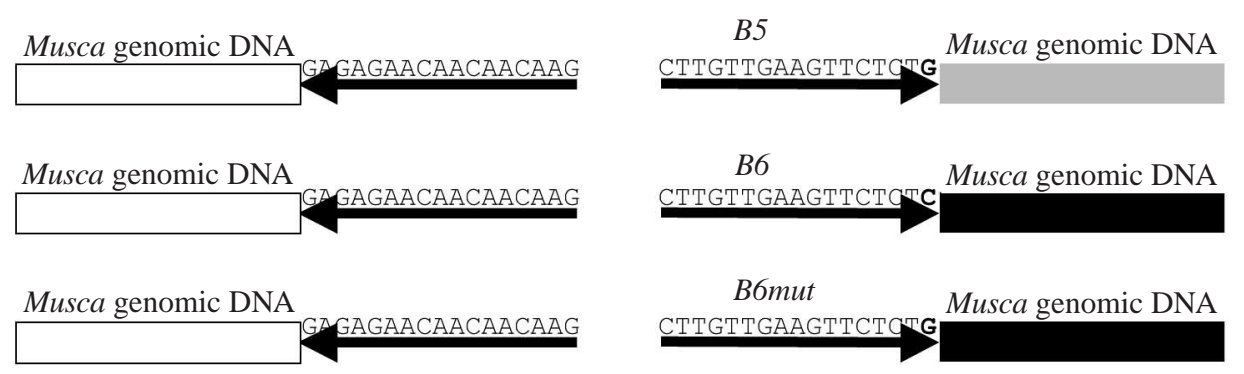

Fig. 1. Comparison of Hermes B5, B6 and B6mut elements. The black arrows represent the inverted terminal repeats (ITRs), and the actual sequences of the ITRs are shown. The terminal nucleotide of the right ITR is highlighted in bold to show the difference between the ends of $B 5$ and B6. The Musca domestica genomic DNA flanking the B5 and B6 elements is different. In the B6mut element, the terminal nucleotide of B6 was changed to a $\mathrm{G}$.

was used in this species. All interplasmid transposition events recovered using the Hermes $B 5$ element were canonical cutand-paste events.

The donor plasmid containing the Hermes B6 element also contains different $M$. domestica genomic DNA at the righthand end of the Hermes element compared with the Hermes $B 5$ donor element. To determine whether the genomic DNA flanking the $B 6$ element was negatively influencing the transposition behavior of the element, the element was converted to a $B 5$ element by changing the terminal $\mathrm{C}$ to a $\mathrm{G}$. This mutated $B 6$ element (B6mut) was identical to the original $B 5$ element, except for this mutation, but contained the flanking genomic DNA next to the $B 6$ element (Fig. 1). When this mutated $B 6$ element was used in transposition assays performed in Drosophila embryos, transpositions of Hermes into the target plasmid were recovered and confirmed both by diagnostic restriction digests and sequencing of the transposition junction fragments with the target plasmid (Table 1). The frequency of transposition was similar to that observed for the $B 5$ form of Hermes, indicating that restoring the terminal nucleotide of Hermes $B 6$ to $\mathrm{G}$ restores the activity of the element. These data also indicate that the $M$. domestica genomic sequence flanking both the $B 5$ and $B 6$ elements plays little or no role in determining the activity of the elements.

The functionality of the Hermes B6 element was tested further in $D$. melanogaster by using it as a germ-line transformation vector following the addition of the mini-white gene from this species. No transgenic individuals were recovered from $71 \mathrm{G}_{0}$ adults and $7532 \mathrm{G}_{1}$ progeny. Previously, we have shown that Hermes B5-mediated transformation of Drosophila occurs at frequencies of approximately 20-60\% (Michel et al., 2001; O'Brochta et al., 1996). Therefore, the failure to obtain germ-line transformants using a $B 6$-containing element indicates that this form of the element is inactive in the Drosophila germ-line.

The mobility properties of Hermes are distinctly altered when introduced into $A$. aegypti. In this species, the sequence requirements for element movement are altered, as indicated by the functionality of the $B 6$ element, and germ-line integration reactions appear to rely on a mechanism other than simple cut and paste. Movements in the soma, as indicated by plasmid-to-plasmid assays, do appear to use a canonical cutand-paste mechanism.

\section{Remobilization}

To follow the fate of Hermes elements in transgenic mosquitoes and to examine their remobilization potential, an autonomous form of the $B 5$ element was constructed (Guimond et al., 2003) and introduced into A. aegypti. As observed for previous germ-line integrations of Hermes in mosquitoes, integration of this element occurred by a mechanism other than cut-and-paste integration. This alternative mechanism resulted in the entire autonomous element being integrated together with sequences on the vector-containing plasmid that flank it

Table 1. Mobility of Hermes B5, B6 and B6mut elements in Drosophila embryos

\begin{tabular}{|c|c|c|c|c|c|c|}
\hline \multirow[b]{2}{*}{ Experiment } & \multirow[b]{2}{*}{ Donor } & \multirow[b]{2}{*}{ Helper } & \multirow{2}{*}{$\begin{array}{c}\text { Target } \\
\text { plasmids } \\
\text { screened }\end{array}$} & \multicolumn{2}{|c|}{ Confirmed transpositions } & \multirow[b]{2}{*}{ Frequency } \\
\hline & & & & Mapped & Sequenced & \\
\hline 1 & B5 & - & 93600 & 0 & & 0 \\
\hline 2 & $B 5$ & + & 76000 & 19 & $2 / 2$ & $0.025 \%$ \\
\hline 3 & B6 & - & 430800 & 0 & & 0 \\
\hline 4 & B6 & + & 738400 & 0 & & 0 \\
\hline 5 & Bбтит & - & 60000 & 0 & & 0 \\
\hline 6 & B6mut & + & 39400 & 14 & $5 / 6$ & $0.036 \%$ \\
\hline
\end{tabular}

Interplasmid transposition assays were performed as described (Sarkar et al., 1997a) using Hermes B5, B6 and B6mut elements, with and without a transposase-encoding helper plasmid. 
A

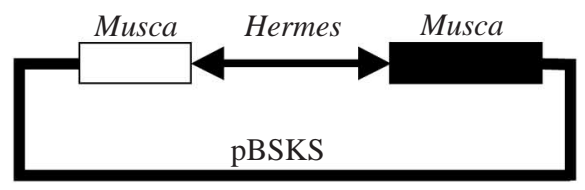

B

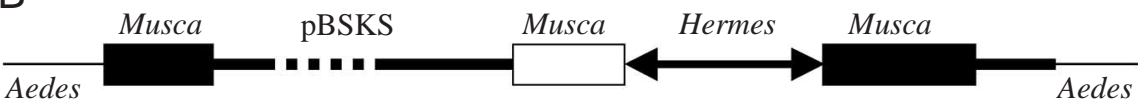

Fig. 2. (A) The structure of plasmid Hermes QH7011 used to genetically transform Aedes aegypti. The plasmid pBSKS contains an autonomous Hermes element with the Hermes transposase gene under the control of the hsp70 promoter of Drosophila melanogaster as well as EGFP (enhanced green fluorescent protein) under the control of the D. melanogaster actin5C promoter (not drawn to scale). M. domestica genomic DNA flanking the ends of Hermes are relics of the original cloning of Hermes and are indicated by boxes. (B) Structure of Hermes QH7011 in the germ line of A. aegypti as deduced by Southern blots and PCR analysis of the breakpoints (data not shown). The entire element has integrated along with the Musca flanking sequences and the pBSKS vector DNA. Rearrangements towards the ends of the entire integrated sequence are shown and consist of a partial duplication of the Musca sequences flanking the right end. In addition, a rearrangement of pBSKS vector DNA in the form of an inversion occurred during the integration process (broken line).

(Fig. 2). This line was inbred for several generations, and transposable element display (TE display) analysis was used to examine the rates and patterns of transposition in this species, as was done with this same element in D. melanogaster (Guimond et al., 2003; Fig. 3). TE display permits the movement of Hermes to be monitored in both the germ line and the soma (Guimond et al., 2003). This method permits each of the unique junction fragments between the right and left ends of the element and flanking genomic DNA to be visualized as a gene amplification product. Transposition events are easily recognized by the amplification of junction fragments, each with a unique molecular mass. The TE display method is also sensitive enough to detect somatic movement of an element. Somatic remobilization during development results in a clone of cells that contain a new genotype with respect to the location of the element. Consequently, an insect in which there is somatic movement of the element as well as germ-line movement will yield a complex pattern of amplification

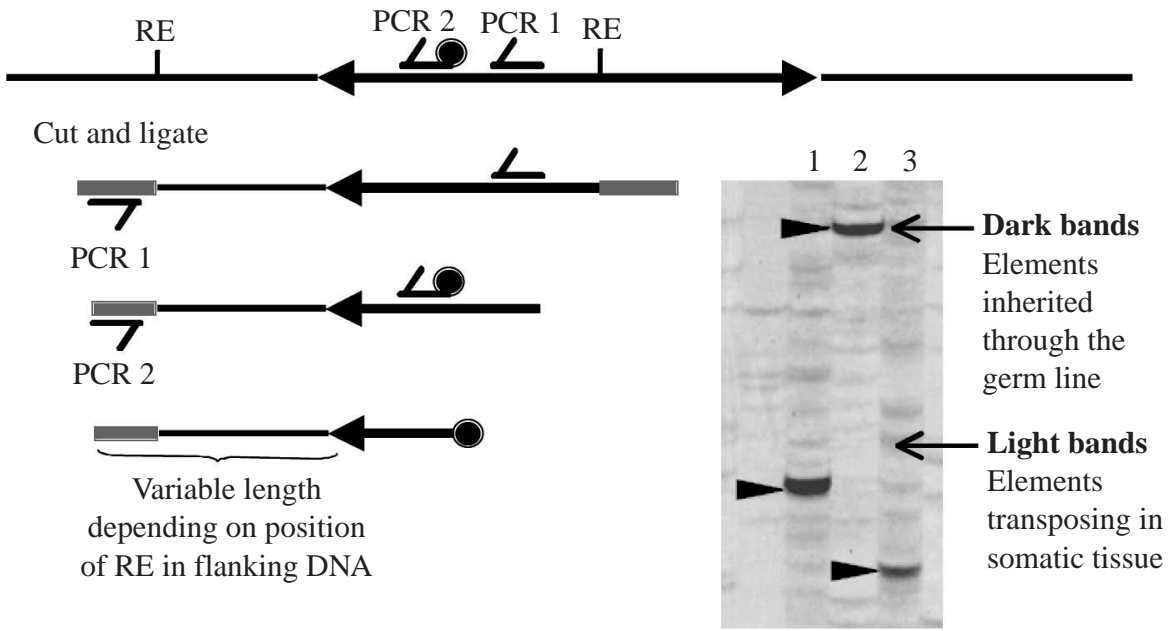

Fig. 3. Summary of the transposable element display method. Genomic DNA is digested with a restriction enzyme (RE) that results in a junction fragment, including the terminal sequences of the element and flanking genomic DNA. Specific adapters are added followed by two rounds of PCR. The first PCR results in the preliminary amplification of the junction fragment, and the second reaction further amplifies the fragments of interest using an element-specific primer labeled with Cy5. Fragments are size fractionated by denaturing acrylamide gel electrophoresis and visualized in a phosphoimager. Each band represents a unique junction fragment. Band intensity reflects template abundance. The most abundant products (darkest bands) are from elements that were inherited vertically, while lighter bands are elements transposing in the somatic tissue of the insect, resulting in clones of cells with the element in a new location. Template abundance of somatic transposition events varies depending on the point in development when transposition occurs. Samples 1-3 represent three Drosophila melanogaster individuals with different genotypes with respect to the location of the autonomous Hermes element inherited through the germ line (arrowheads). 
products following TE display (Fig. 3). Elements inherited vertically through the germ line will be present in all cells of the insect (if there is no excision) and will yield an abundance of template from which amplification products will arise, while those elements transposing somatically will result in relatively less abundant template and products. In a TE display, somatic transposition events are seen as less-abundant PCR products relative to those arising from the vertically inherited element (Guimond et al., 2003). Somatic remobilization results in an insect that is a mosaic of genotypes. Somatic transpositions occurring early in development will be abundantly represented in the pool of template DNA, while those arising late will be under-represented. Somatic transpositions will therefore yield PCR products in the TE display method with varying abundance. As shown in Fig. 4A, Hermes B5 is clearly mobile in somatic nuclei in this transgenic line of A. aegypti. Only a few weakly labeled fragments were seen in both wild-type untransformed mosquitoes and in transgenic mosquitoes containing the Hermes element into which the actin5C:EGFP (enhanced green fluorescent protein) gene but not the Hermes transposase gene had been inserted. Since the latter does not encode its own transposase, it is incapable of mediating its own movement through the A. aegypti genome.

A number of well-isolated bands were excised, cloned, and their sequence determined, verifying the presence of Hermes terminal sequences and unique flanking DNA resulting from the transposition of the parental element (Fig. 4). In some cases, BLASTX analysis revealed similarities to sequences from gag proteins (L15 in Fig. 4) and integrase proteins (L16 in Fig. 4) encoded by retroviruses. The $8 \mathrm{bp}$ of sequence immediately flanking the Hermes ITR displayed similarity to the $8 \mathrm{bp}$ consensus target-site duplication (5'-GTnnnnAC-3') characterized for other $h A T$ elements. The presence of these fragments indicates the transposition of the autonomous Hermes element in new sites in the genome of A. aegypti somatic cells. Similar sequences indicative of cut-and-paste transposition of the Hermes element in these mosquitoes were also obtained from TE display analysis of the left-hand Hermes end (Fig. 4).

These data demonstrate that an autonomous Hermes element is capable of transposing in the soma of A. aegypti and does so in a way that is typical of Class II transposable elements. Following the initial integration of Hermes into the germ line of A. aegypti, this element becomes highly stable. By contrast, despite repeated efforts, we have never been able to detect the subsequent remobilization of this autonomous Hermes element in the germ line of $A$. aegypti. Thus, despite the transposase being placed under the control of the hsp70 promoter, remobilization of this autonomous Hermes element differs both between Drosophila and Aedes germ lines and between the
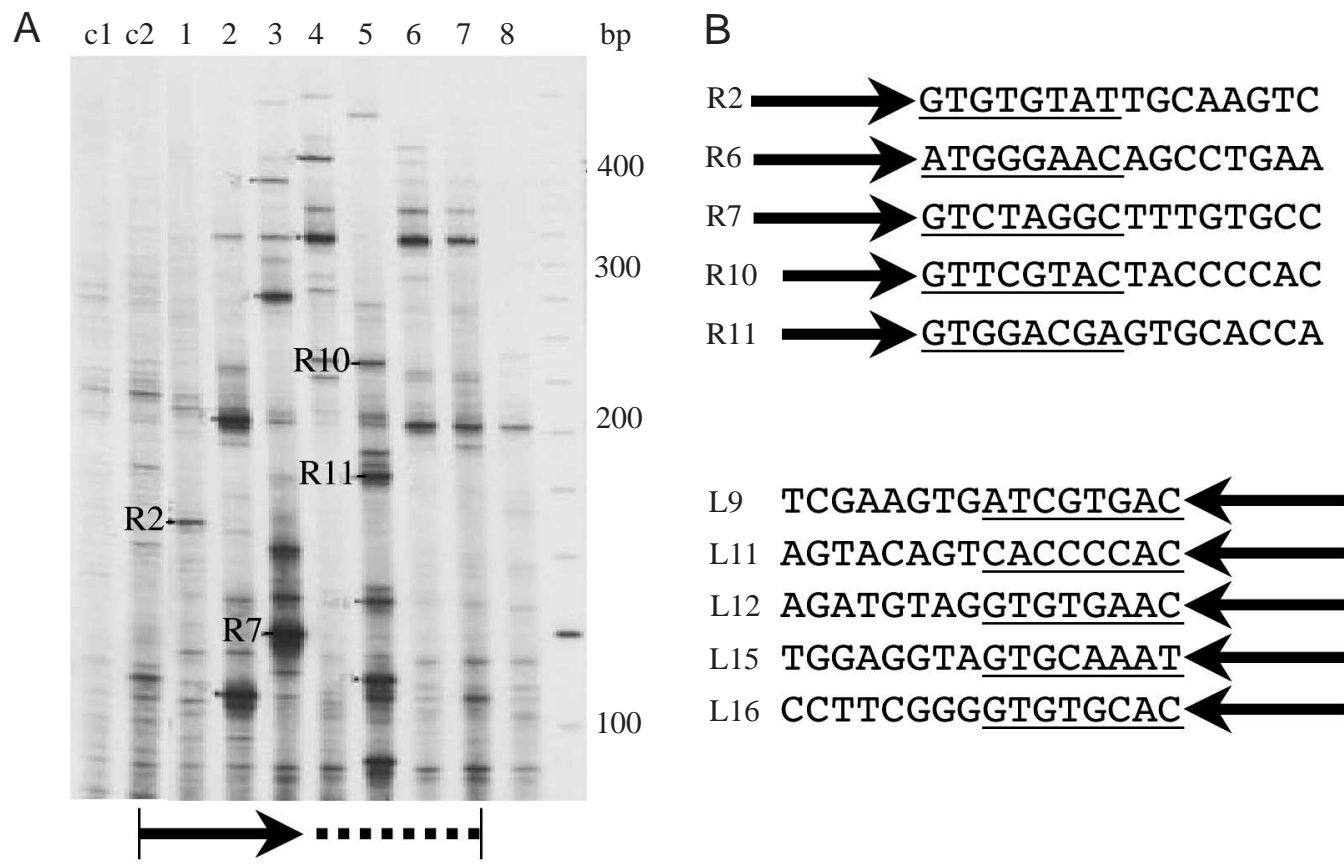

200

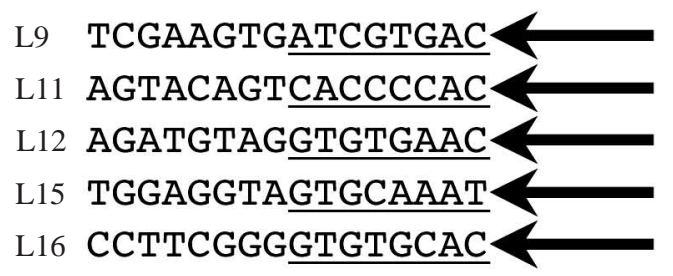

Fig. 4. (A) Transposable element display analysis visualizing Hermes right-end junction fragments in individual Aedes aegypti containing the autonomous Hermes QH7010 element. c1 and c2 are controls: c1 is a non-transgenic wild-type mosquito and c2 is a transgenic mosquito containing a non-autonomous Hermes element also containing the actin5C:EGFP gene (Pinkerton et al., 2000). Bands in c1 and c2 are considered non-specific PCR products. Multiple, intensely labeled fragments were observed only from DNA prepared from the individuals containing the autonomous element. Molecular size markers, in base pairs, are shown. Bands isolated, reamplified and sequenced from this experiment are indicated (R2, R7, R10 and R11). (B) Labeled fragments were excised from transposable element display gels containing leftand right-hand Hermes ends (left-hand analysis not shown here), and their sequences were determined. Hermes inverted terminal repeat (ITR) sequences are indicated by the black arrows, and flanking $A$. aegypti sequences are shown with the proposed 8 bp target site duplications underlined. Only partial flanking sequences are shown, i.e. those immediately adjacent to the Hermes ITRs. 
soma and germ line of Aedes, indicating that regulation of Hermes transposition is controlled to some degree posttranscriptionally.

\section{mariner \\ Transposition}

The Mos 1 mariner element (Mos1) from D. maurutiana has a very broad host range and has been used to create transgenic microbes, protozoans, insects and vertebrates (Lampe et al., 2000). Consistent with these empirical findings of widespread functionality is the distribution pattern of mariner elements in the genomes of plants and animals. More than $15 \%$ of the 400 insect species examined for the presence of mariner elements tested positive (Robertson, 1993). In some cases, the presence of the element within a species seems best explained by horizontal transfer from another organism by an unknown mechanism followed by element amplification by transposition. These data suggest that mariner elements are autonomous recombination systems requiring few or no host factors for their movement. In vitro studies with purified transposase, donor elements and target sequences seem to support this conclusion, since transposition of Mos 1 occurs in vitro in only the presence of transposase, $\mathrm{Mg}^{2+}$ and target DNA (Lampe and Robertson, 1996).

Plasmid-based mobility assays in developing insect embryos demonstrated the ability of Mosl to function in A. aegypti as well as in other insects (Coates et al., 1995, 1997). Transposition followed the typical pattern of cut and paste and resulted in the creation of characteristic TA target-site duplications. Mos 1 was used to create transgenic insects by Lidholm et al. (1993), who successfully transformed $D$. melanogaster using Mosl-based vectors carrying the D. melanogaster mini-white gene. While Mos 1 functioned as a gene vector in this species, the rate of integration was considerably lower (approximately 5\%) than that observed when $P$, Hermes and hobo elements were used as vectors in this species. Integrations of Mos 1 into the germ line of $D$. melanogaster were always by the canonical cut-and-paste mechanism. Mosl has also been used repeatedly to create transgenic A. aegypti, beginning with Coates et al. (1998), who observed a rate of integration of approximately $4 \%$. During the transformation of A. aegypti, Coates et al. (1998) found that three of the four initial lines contained Mos 1 elements that had integrated in a cut-and-paste manner; however, one of the lines contained the Mos 1 element together with flanking plasmid DNA sequences, similar to what is routinely seen following Hermes integration in the germ line of this species. Germ-line transformation of A. aegypti using Mosl can also be achieved by co-injecting a $M o s 1$ vector and purified Mos 1 transposase protein instead of a helper plasmid (Coates et al., 2000). Interestingly, in this experiment, all (7/7) of the integration events involved not only the Mos 1 element but also sequences flanking the element on the original vector-containing plasmid. Therefore, under some conditions, the integration behavior of Mos 1 resembles the Hermes element in that transpositional recombination appears to lose some fidelity and the ITRs fail to accurately delimit the sequences involved in the reaction.

The Himarl element, reconstructed from the closely related Cpmarl element from Chrysoperla plorabunda using a deduced consensus sequence of a mariner-like element in $H$. irritans, also shows some unexpected host-dependent behavior. Although this element is functional in vitro and in a wide range of prokaryotes, it appears to be completely immobile in insects (Lampe et al., 2000). Robust efforts to create transgenic $D$. melanogaster by embryo microinjection have repeatedly failed and are generally consistent with the observations of others using Mosl as a primary transformation vector. Even mutants of the Himarl element isolated from Escherichia coli that are hyperactive in microbes are immobile in insects, suggesting that activity per se appears not to be the factor limiting or preventing the activity of this element in insects (Lampe et al., 2000).

\section{Remobilization}

The remobilization potential of existing Mos 1 -based gene vectors is very low in both $D$. melanogaster and A. aegypti. Lidholm et al. (1993) reported somatic mosaicism in $\leq 1 \%$ of the progeny containing a Mos 1 vector. This is in stark contrast to the extreme instability of the white peach $\left(w^{p c h}\right)$ allele of $D$. mauritiana, which contained the first-isolated mariner element. The mariner element present in $w^{p c h}$ was active in the presence of Mosl transposase and resulted in somatic mosaicism in $100 \%$ of the progeny. Consequently, the high degree of stability of the Mos 1 vectors used by Lidholm et al. (1993) was quite unexpected. Other descriptions of the postintegration stability of Mosl vectors followed (Lohe et al., 1995; Lozovsky et al., 2002). Lozovsky et al. (2002) reported testing six Mos 1 vectors in which the transgenic marker gene varied in length and position within the vector. Following the introduction of these elements into D. melanogaster using Hermes vectors, attempts were made to remobilize the Mos 1 elements by providing functional transposase in trans. Of the four vectors that permitted somatic movement to be assessed, only one showed any evidence of somatic movement based on mosaicism in the eye and in this case at a rate of $0.23 \%$. Using more-sensitive PCR-based methods to detect Mos 1 movement (excision) throughout the body, only two of the four vectors tested displayed evidence of somatic movement. The other two vectors tested appeared to be completely immobile following their introduction into D. melanogaster.

Evidence for Mos1 remobilization in A. aegypti was first obtained by injecting embryos from transgenic lines containing cinnabar (cn)-marked Mos 1 vectors with plasmids expressing Mos1 transposase (N.J., C.J.C. and A.A.J., unpublished data). Adults developing from injected embryos were backcrossed to $k h^{w}$, and progeny with pigmented eyes different from the parental phenotype were considered possible products of transposition. Of the 24413 progeny with pigmented eyes examined, 2938 had phenotypes distinguishable from the parentals. A sample of these exceptional progeny was used to establish 24 families whose progeny was examined by 


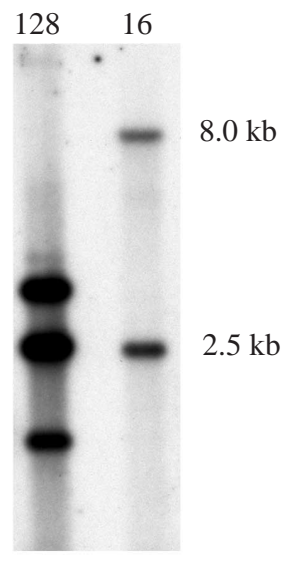

Fig. 5. Southern blot analysis of a line derived from a germ-line transposition of a cn-carrying Mos 1 vector. Line 16 was started from one individual whose parent was from line 128 and injected with a helper plasmid expressing before blastoderm formation. One of the resulting progeny had an eye color different from the parental insects and was used to establish line 16. Genomic DNA was isolated from adults and cut with $S a c I$, which cuts twice within the gene vector, and transferred to a nylon membrane. The filter was hybridized with a radiolabeled $c n+$ gene fragment (see fig. 1 of Coates et al., 1998 for details of the analysis). An internal $2.5-\mathrm{kb}$ fragment is present in lines 128 and 16. Additional hybridizing fragments are diagnostic of independent insertion sites within the genome. The difference in pattern between 128 and 16 indicates the presence of a transposition event.
Southern blot analysis. One line contained the Mos 1 vector in a new location, indicating that a transposition event had occurred and demonstrating the potential of Mosl to be remobilized in this species (Fig. 5).

An alternative strategy for testing Mos 1 remobilization was used by Wilson et al. (2003). Two transgenic lines were created using Mosl vectors containing the $c n$ gene from $D$. melanogaster in an A. aegypti strain homozygous for $k h^{w}$. To examine the remobilization potential of these elements in $A$. aegypti, heterozygotes were created with a third transgenic line containing a piggyBac vector into which Mos 1 transposase gene under the regulatory control of the hsp 82 promoter of Drosophila pseudoobscurra had been placed. Progeny from these heterozygotes was examined, and red-eyed progeny with an eye color phenotype different from the heterozygote parental insects was selected as putative transposition events and analyzed further using TE display (Guimond et al., 2003). Of the 14000 red-eyed progeny examined by Wilson et al. (2003), only one contained a remobilized Mos 1 vector (Fig. 6). These investigators also tested for somatic mobility of the Mosl elements by performing TE display on Mos 1-cn/hspMos 1 heterozygotes. Approximately 50\% of the 30 individual heterozygotes analyzed had evidence of at least one somatic remobilization event. In those individuals that had evidence for somatic movement, fewer than five events were detected. Wilson et al. (2003) did not estimate a frequency of somatic transposition but it appeared to be at least 10-fold lower than

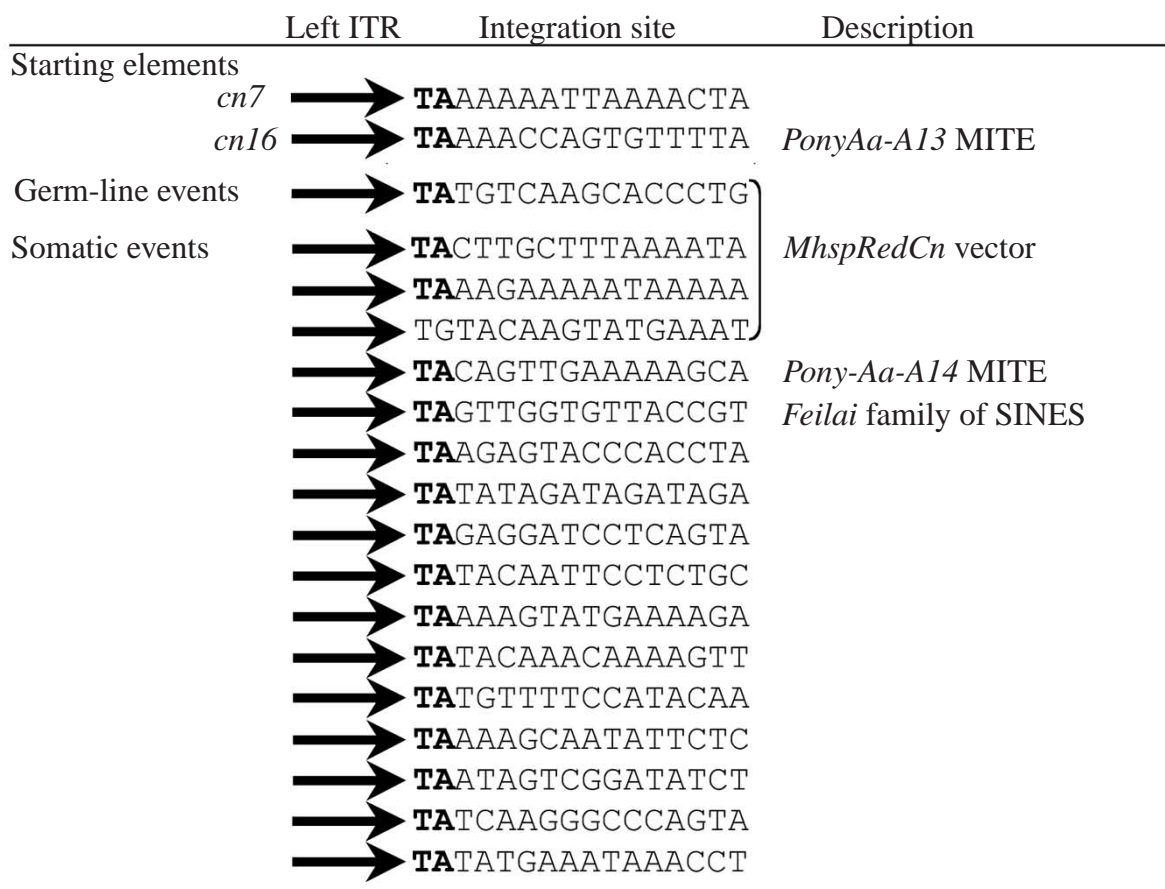

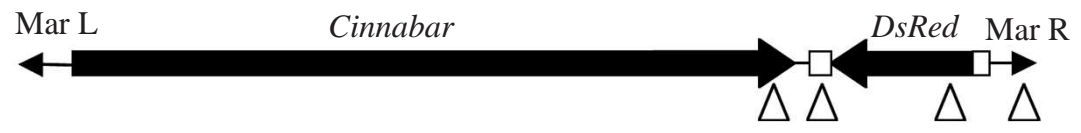

Fig. 6. (A) Germ-line and somatic transpositions of Mos1 in Aedes aegypti (based on data reported by Wilson et al., 2003). Black arrows represent the terminal sequences at the left end of Mosl. The sequences of the integration sites and, where it is known, the name of the locus into which the element integrated are shown. Four of the integration events were into the original Mos1 vector, and the location of these transposition events within the vector is indicated by open triangles below the diagram of the Mos 1 vector. Mar L and Mar $\mathrm{R}$ refer to the inverted terminal repeats (ITRs) of the Mos 1 vector. Cinnabar and DsRed are transgenes contained on the vector. Cinnabar was used as a transformation marker and DsRed was part of an enhancer-reporter system. 
that observed with an autonomous Hermes element in $D$. melanogaster (Guimond et al., 2003).

The stability of Mos 1 in A. aegypti was similar to that described in D. melanogaster by others (Lidholm et al., 1993; Lohe et al., 1995; Lozovsky et al., 2002). The unusual postintegration stability of Mos 1 in D. melanogaster appears not to be a specific host effect but instead appears to be a general characteristic of the Mos 1 element and observed in all species of insects into which it is inserted.

The basis of this post-integration stability remains unknown, although the current model proposes that there are critical sequences and spacing of sequences within Mosl that are important in determining the efficiency of movement (Lohe and Hartl, 2002). Lozovsky et al. (2002) found only one element that could be remobilized at detectable levels, and this particular vector consisted of almost two complete elements oriented as direct repeats and each flanking the marker gene. These investigators suggested that there are critical spacing requirements between certain sequence components of the element. Inserting marker genes and transgenes disrupts this spacing and impairs the element's ability to remobilize.

Lohe and Hartl (2002) also suggest that Mos1 movement in insects is dependent upon the presence of at least three blocks of sequences located throughout the element. These critical sequences are required in cis and are located at least $200 \mathrm{bp}$ from the ends of the element. The 'critical sequence' hypothesis of Lohe and Hartl (2002) is somewhat contentious for two reasons. First, while others have described the role of sequences other than the ITRs in the transposition process of other elements, these sequences have always been immediately sub-terminal and not dispersed throughout the element as proposed by these investigators. In addition, the cis-sequence requirements of Mos 1 have been determined in vitro, and only approximately $60 \mathrm{bp}$ and $30 \mathrm{bp}$ of the left and right ends, respectively, of the element are essential for in vitro transposition activity of a $1.1 \mathrm{~kb}$ vector (Tosi and Beverly, 2000). The minimized element created by Tosi and Beverly (2000) was only twofold less active than an unmodified element under the same conditions. Clearly, the data of Lohe and Hartl (2002) and Tosi and Beverly (2000) indicate an influence of the host on the activity and behavior of the element that is consistent with the observed immobility of functional and hyperactive Himarl elements in insects.

The post-integration behavior of Mos1 in A. aegypti also revealed an aspect of this element that could be relevant to future applications. Of the somatic and germ-line Mos 1 remobilization events recovered and analyzed by Wilson et al. (2003), approximately 25\% (4/17) were instances of where the element transposed into a copy of itself. In all of the experiments in their study, the element was in a heterozygous condition, and therefore only during mitosis in diploid cells is a second copy of the element present to serve as a target for Mos 1 transposition. It therefore appears that Mos 1 can transpose sometime between the beginning of $S$ phase and the end of metaphase. Transposition during $\mathrm{S}$ phase has been reported for the $A c / D s$ element of maize and it is thought to play an important role in the increase in element copy number over time. Because cut-and-paste transposition is a conservative mechanism leading to no net increase in copy number, Class II elements must exploit in some way the host replicative machinery in order to increase in copy number within a genome. Replication may rely on gap repair mechanisms following element excision (Gloor et al., 1991; Lohe et al., 2000; Nassif et al., 1994) or by timing transposition to occur during chromosome replication (Fedoroff, 1989; Greenblatt and Brink, 1963; Wilson et al., 2003). The remobilization events reported by Wilson et al. (2003) may have taken place in polyploid cells in which there would be many copies of the Mos 1 element to serve as targets. Hence, the somatic transposition events detected, isolated and analyzed by TE display would each have come from a single polyploid cell. It is not clear whether the method of TE display has the level of sensitivity that would permit the detection of transposition events represented by perhaps only hundreds of copies of the template. In any case, however, the pattern of transposition of Mos1 indicates a distinctly nonrandom distribution. Preferential integration of the element into copies of itself on sister chromatids or homologous chromosomes suggests that the element may have a propensity to undergo local hopping whereby an element is more likely to jump to linked sites, often only kilobases or less from the initial integration site. Furthermore, the targeting of the element itself reflects a bias in the target site selection process that is similar to that seen during the process of element homing in which sequences contained on the element direct the transposition process to homologous sequences. Additional experiments would be needed in order to specifically address these hypotheses.

\section{piggyBac \\ Transposition}

piggyBac vectors have been used to transform a wide range of insects, including representatives from the orders Diptera, Lepidoptera, Coleoptera and Hymenoptera. The Diptera have included the mosquitoes A. aegypti (Kokoza et al., 2001; Lobo et al., 2002), Anopheles stephensi (Nolan et al., 2002), Anopheles albimanus (Perera et al., 2002) and Anopheles gambiae (Grossman et al., 2001), with transformation efficiencies ranging from approximately $1 \%$ in $A$. gambiae to $40 \%$ in A. albimanus. Initial reports described all piggyBac integrations in mosquitoes as arising from perfect cut-andpaste transposition reactions into TTAA target sites that were duplicated during integration. More recently in A. aegypti, exceptional transposition events have been recovered and described. Adelman et al. (in press) created six transgenic lines of A. aegypti containing piggyBac vectors with EGFP under the regulatory control of the $3 X P 3$ promoter. Two of the four lines analyzed further arose from the insertion of a single vector. The remaining two lines arose from the insertion of multiple copies of the piggyBac vector in tandem arrays. In addition, these complex integrations of the piggyBac vector 
were accompanied by the incorporation of helper-plasmid sequences containing piggyBac transposase. These transgenics appeared to arise from either the non-canonical transposition of piggyBac or by recombination mechanisms unrelated to transposition.

After screening the progeny of approximately $900 \mathrm{G}_{0} A$. aegypti adults homozygous for the $k h^{w}$ allele, we recovered four independent transgenic lines expressing the kynurenine hydroxylase gene ( $c n$ ) from D. melanogaster (D.A.O'B., N.S. and P.W.A., unpublished data). Two of these lines resulted from cut-and-paste transposition events in which only sequences delimited by the ITRs transposed. The remaining two lines arose from non-canonical reactions and resulted in the transposition of sequences flanking the element on the original donor plasmid. These events resembled Hermes integration events in this species, in which flanking DNA present on the donor plasmid is invariably transferred during the transpositional recombination reaction in the germ line during transformation. In both cases, only a single element integrated and, while the extent of the flanking DNA has not been fully determined, it includes at least $200 \mathrm{bp}$ of vector sequences at both ends.

\section{Remobilization}

Post-integration remobilization of piggyBac has been reported in D. melanogaster (Horn et al., 2003) and Tribolium castaneum (Lorenzen et al., in press). Horn et al. (2003) described the remobilization of piggyBac by establishing transgenic lines of $D$. melanogaster containing a single insertion of a non-autonomous piggyBac vector containing enhancer-reporter genes (reporter lines) and other lines containing single insertions of either a Hermes, mariner or Minos element containing the piggyBac transposase gene under the regulatory control of a strong promoter (jumpstarter lines). Remobilization was induced by crossing reporter and jumpstarter lines and testing the progeny for reporter elements with new linkage relationships relative to the original element. These investigators found that, on average, $80 \%$ of the heterozygotes tested produced progeny that included a transposition event. All transposition events were canonical cut-and-paste recombination reactions. For T. castaneum, remobilization was achieved by injecting developing embryos from a transgenic line containing a single piggyBac element containing a unique reporter gene expression pattern $(3 x P 3$ $E G F P$ ) resulting from the local influence of a tissue-specific enhancer with a piggyBac transposase-expressing helper plasmid. $\mathrm{G}_{1}$ individuals were screened for a modified reporter gene expression pattern. Of the 32 adults developing from embryos injected with helper plasmid, nine produced progeny that had an altered pattern of reporter gene expression relative to the parental insects, yielding an estimated rate of remobilization under these conditions of $28 \%$.

Currently, there are no well-documented cases of piggyBac remobilization in mosquitoes. Adelman et al. (in press) observed what appeared to be severe instability of the arrays of piggyBac vectors in two of their transgenic lines. These arrays appeared capable of wholesale excision and loss in one generation. The role of piggyBac transposase in this process is not known. These investigators report no evidence for piggyBac transposition within the genome of these transgenics despite the presence of a piggyBac transposase source. We have constructed multiple reporter and jumpstarter lines of $A$. aegypti homozygous for $k h^{w}$ (D.A.O'B., N.S. and P.W.A., unpublished data). Reporter lines were created using a nonautonomous piggyBac vector expressing the wild-type $D$. melanogaster cinnabar (cn) gene and also containing DsRed under the minimal, un-enhanced $h s p 70$ promoter of $D$. melanogaster. The DsRed gene in this vector serves as a reporter of local enhancer activity. The piggyBac vector can serve as a reporter of remobilization in two ways. First, $c n$ gene expression in transgenic A. aegypti is position dependent and will allow transposition events to be recovered by selecting progeny with an altered eye color phenotype. Second, the appearance of a novel pattern of DsRed expression in progeny relative to the parental line will indicate the relocation of the reporter element within the genome of the progeny. Jumpstarter lines were created using non-autonomous mariner vectors containing EGFP under the regulatory control of the $3 X P 3$ promoter and the piggyBac transposase gene under the regulatory control of the $D$. melanogaster $h s p 70$ promoter. The hsp::pBactransposase gene in the jumpstarter element was the same gene contained on the helper plasmid used in the creation of the initial piggyBac-containing reporter lines. Heterozygous insects containing combinations of the piggyBac remobilization reporter elements and jumpstarter lines were created and backcrossed to $k h^{w}$. Progeny expressing $c n$ and with a phenotype different from the heterozygous parent were selected as potential remobilization events. The genotype of these insects was determined by TE display using piggyBacspecific primers. To date, none of the A. aegypti progeny selected on the basis of an altered eye color phenotype (putative remobilizations) have yielded a pattern of PCR products from TE display that is consistent with germ-line or somatic movement of the element (Fig. 7). Only a limited number (3000) of progeny have been screened and 100 of these have been genotyped by TE display because they had an eye color phenotype that suggested the presence of a transposition event. The 3000 progeny screened arose from a cross between $k h^{w}$ and heterozygotes for reporter and jumpstarter elements that had been heat-shocked once during larval development at $41^{\circ} \mathrm{C}$ for one hour. Under the limited conditions and scope of this experiment, piggyBac does not appear to be efficiently remobilized in A. aegypti.

\section{Minos \\ Transposition}

Minos, although tested less extensively than other insect gene vectors, appears to have a broad host range. Evidence for transposition activity in Orthoptera, Lepidoptera and Diptera, including the mosquito A. stephensi (Catteruccia et al., 2000a,b; Klinakis et al., 2000; Zhang et al., 2002), has been 


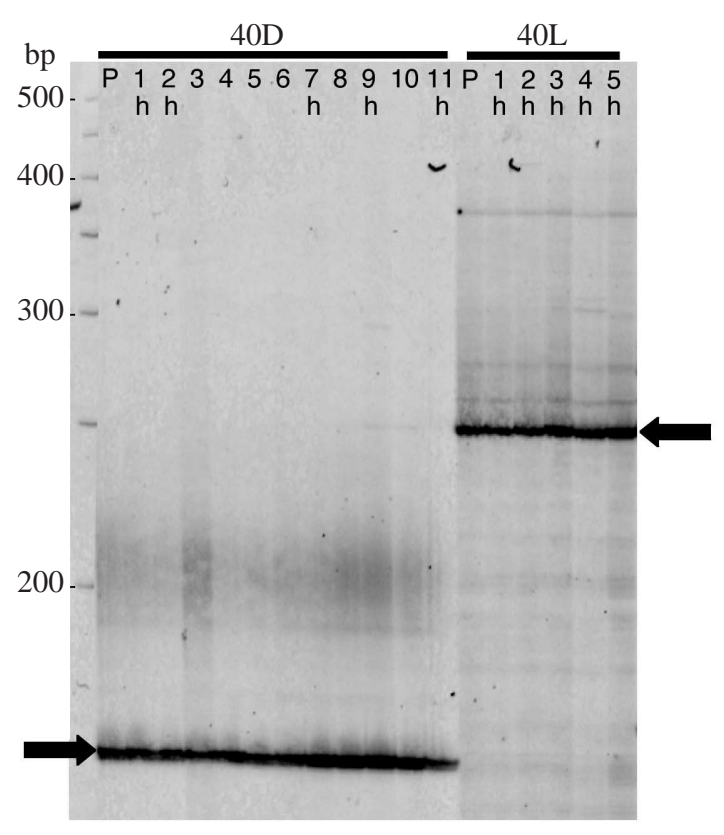

Fig. 7. Transposable element display analysis of progeny arising from a cross between individuals heterozygous for a nonautonomous piggyBac element and a Mos1 vector containing the piggyBac transposase gene under the regulatory control of the $h s p 70$ promoter of Drosophila melanogaster and individuals homozygous for $k h^{w}$. These progeny were selected for analysis because their eye color phenotype was different from the parental phenotype, suggesting that a transposition resulting in a position-dependent alteration in the phenotype had occurred. Progeny from two parental lines are shown (40D, 40L). The piggyBac element inherited through the germ line yields an intensely labeled PCR product (arrow). P refers to the parental insect. Numbered lanes contain results from individual progeny. No evidence of germ-line movement is present. Some of the progeny analyzed were themselves heterozygous for the non-autonomous element and the transposase-expressing Mos 1 vector (h). In these heterozygotes, there was no evidence for somatic transposition of the elements. Molecular size markers, in base pairs, are shown.

reported. Recently, evidence for its ability to transpose in mammalian cells was reported (Drabek et al., 2003). Germ-line transposition rates in insects are similar to those reported for other elements. In the medfly (Ceratitis capitata), transgenics were recovered from $1-3 \%$ of the fertile $\mathrm{G}_{0}$ adults (Loukeris et al., 1995) and this frequency can be improved by directly providing transposase mRNA during the embryo injection process (Kapetanaki et al., 2002). In all cases where it has been determined, Minos integrates using a cut-and-paste mechanism and there is no evidence for non-canonical integration events as reported for mariner, Hermes and piggyBac.

\section{Remobilization}

There are no published data regarding the ability of Minos to be remobilized following its integration into the genome of any organism. Unpublished claims of efficient remobilization of Minos in D. melanogaster exist; however, the data supporting these claims have not been published (http://www.minosbiosystems.com/). Similarly, preliminary attempts have been made to remobilize integrated Minos elements in A. stephensi. Heterozygotes were created by crossing a Minos-containing vector and a line expressing Minos transposase. No progeny that had the Minos element in a new position as a result of transposition was recovered from these insects, indicating that under the conditions tested by the investigators remobilization did not occur (F. Catteruccia and A. Crisanti, Imperial College, personal communication).

\section{Discussion}

Limited data currently exist about the remobilization potential of existing insect gene vectors after their integration into a foreign genome. Most evidence has been obtained from experiments with $D$. melanogaster and has shown that Hermes and piggyBac remobilization occurs readily in this species. The rates of remobilization of Hermes and piggyBac differ, although other aspects of the behavior of these systems, such as integration site specificity with respect to chromosomal regions and genes, have not been systematically compared. Mos 1 stands in contrast to these elements in that it is almost immobile following its initial integration into the genome of D. melanogaster. Mos 1 appears to contain more stringent size requirements as well as constraints on the presence and spacing of cis-critical sequences.

The behavior of current gene vectors in D. melanogaster does not necessarily reflect the behavior of these elements in mosquito genomes. Whereas Hermes, Mos 1 and piggyBac are functional in mosquitoes, certain differences in behavior can be seen, both among these elements and between $D$. melanogaster and mosquitoes. All of the elements appear to have similar activities as primary transformation vectors in, for example, A. aegypti and yield transformants from $2-5 \%$ of the surviving injected embryos. All three elements have displayed non-canonical transposition behavior during the primary integration reaction. In the case of Hermes, all primary integration events involve a mechanism other than cut-andpaste transposition. Mos 1 and piggyBac display cut-and-paste transposition activity, and evidence also exists for a noncanonical transposition reaction similar to that seen with Hermes. Although the nature of this alternative mechanism(s) is not known, it consistently results in the transposition and integration of non-vector sequences that flank the ITRs of the element in the donor plasmid. None of the elements, under the conditions tested, appears to remobilize efficiently in the germ line of this species. Only very rare germ-line transposition events have been detected with Mos1. Hermes and piggyBac remobilizations in the germ line have not been detected. However, Hermes and Mos 1 do appear to remobilize in the soma of A. aegypti. Although the rates of somatic transposition are unknown, it is clear that for both species the remobilization events involve cut-and-paste transposition reactions. Currently, we have no evidence of somatic activity of piggyBac in the soma of A. aegypti. The behavior of Minos, 
although less well characterized, seems to resemble that of the other elements, with evidence for remobilization in $D$. melanogaster but not in mosquitoes.

The post-integration behavior of Hermes, Mos1, Minos and piggyBac is revealing interesting aspects of each of the elements and of the species in which they are being tested. First, these studies illustrate that there can be significant interactions between the transposable element and the host. Even though a transposable element system can be shown to function with minimal requirements in vitro, consisting of only transposase, $\mathrm{Mg}^{2+}$ and target DNA, the requirements for proper functioning in vivo may be more extensive. These studies also illustrate how different elements can be affected more or less by the same factors. Finally, it appears notable that the 'unusual' behaviors of the existing insect gene vectors are pronounced in the germ line of A. aegypti and apparently not in the germ line of other insects. While more work needs to be done to explore the post-integration behavior of these elements, it is interesting to speculate that A. aegypti may possess a mechanism in its germ cells that helps it resist invasion by horizontally transferred DNA.

On a practical level, the apparent stability of the vectors tested in the germ line of $A$. aegypti indicates that the stability of the transgenics being created with these insects will be very high even in the presence of homologous functional transposase. These observations may be significant in assessing the risks associated with the creation and eventual release of transgenic mosquitoes into the environment.

The authors' research reported here was supported in part by the National Institutes of Health and The Wellcome Trust.

\section{References}

Adelman, Z. N., Jasinskiene, N., Vally, K. J. M., Peek, C., Travanty, E. A., Olson, K. E., Brown, S. E., Stephens, J. L., Knudson, D. L., Coates, C. J. et al. (in press). Phenotypic and genotypic instability of EGFP expression in the yellow fever mosquito, Aedes aegypti, following transformation with the piggyBac transposable element. Genetics.

Allen, M., O'Brochta, D. A., Atkinson, P. W. and LeVesque, C. S. (2001). Stable germ-line transformation of Culex quinquefasciatus (Diptera: Culicidae). J. Med. Entomol. 38, 701-710.

Bryan, G. J., Jacobson, J. W. and Hartl, D. L. (1987). Heritable somatic excision of a Drosophila transposon. Science 235, 1636-1638.

Catteruccia, F., Nolan, T., Blass, C., Muller, H. M., Crisanti, A., Kafatos, F. C. and Loukeris, T. G. (2000a). Toward Anopheles transformation: Minos element activity in anopheline cells and embryos. Proc. Natl. Acad. Sci. USA 97, 2157-2162.

Catteruccia, F., Nolan, T., Loukeris, T. G., Blass, C., Savakis, C., Kafatos, F. C. and Crisanti, A. (2000b). Stable germline transformation of the malaria mosquito Anopheles stephensi. Nature 405, 959-962.

Coates, C. J., Jasinskiene, N., Miyashiro, L. and James, A. A. (1998). Mariner transposition and transformation of the yellow fever mosquito, Aedes aegypti. Proc. Natl. Acad. Sci. USA 95, 3748-3751.

Coates, C. J., Jasinskiene, N., Morgan, D., Tosi, L. R. O., Beverly, S. M. and James, A. A. (2000). Purified mariner (Mos1) transposase catalyzes the integration of marked elements into the germ-line of the yellow fever mosquito, Aedes aegypti. Insect Biochem. Mol. Biol. 30, 1003-1008.

Coates, C. J., Truney, C. L., Frommer, M., O'Brochta, D. A., Warren, W. D. and Atkinson, P. W. (1995). The transposable element Mariner can excise in non-drosophilid insects. Mol. Gen. Genet. 249, 246-252.

Coates, C. J., Turney, C. L., Frommer, M., O'Brochta, D. A. and Atkinson, P. W. (1997). Interplasmid transposition of the mariner transposable element in non-drosophilid insects. Mol. Gen. Genet. 253, 728733.

Drabek, D., Zagoraiou, L., de Wit, T., Langeveld, A., Roumpaki, C., Mamalaki, C., Savakis, C. and Grosveld, F. (2003). Transposition of the Drosophila hydei Minos transposon in the mouse germ line. Genomics 81, 108-111.

Fedoroff, N. V. (1989). Maize Transposable Elements. Washington, DC: American Society for Microbiology.

Gloor, G. B., Nassif, N. A., Johnson-Schlitz, D. M., Preston, C. R. and Engels, W. R. (1991). Targeted gene replacement in Drosophila via $P$ element-induced gap repair. Science 253, 1110-1117.

Greenblatt, I. M. and Brink, R. A. (1963). Transposition of Modulator in maize into divided and undivided chromosome segments. Nature 292, 860862.

Grossman, G. L., Rafferty, C. S., Clayton, J. R., Stevens, T. K., Mukabayire, O. and Benedict, M. Q. (2001). Germline transformation of the malaria vector, Anopheles gambiae, with the piggyBac transposable element. Insect Mol. Biol. 10, 597-604.

Guimond, N., Bideshi, D. K., Pinkerton, A. C., Atkinson, P. W. and O'Brochta, D. A. (2003). Patterns of Hermes transposition in Drosophila melanogaster. Mol. Gen. Genet. 268, 779-790.

Horn, C., Offen, N., Nystedt, S., Haecker, U. and Wimmer, E. A. (2003). piggyBac-based insertional mutagenesis and enhancer detection as a tool for functional insect genomics. Genetics 163, 647-661.

Jasinskiene, N., Coates, C. J., Benedict, M. Q., Cornel, A. J., Rafferty, C., Salazar-Rafferty, C., James, A. A. and Collins, F. H. (1998). Stable, transposon mediated transformation of the yellow fever mosquito, Aedes aegypti, using the Hermes element from the housefly. Proc. Natl. Acad. Sci. USA 95, 3743-3747.

Jasinskiene, N., Coates, C. J. and James, A. A. (2000). Structure of Hermes integrations in the germline of the yellow fever mosquito, Aedes aegypti. Insect Mol. Biol. 9, 11-18.

Kapetanaki, M. G., Loukeris, T. G., Livadaras, I. and Savakis, C. (2002). High frequencies of Minos transposon mobilization are obtained in insects by using in vitro synthesized mRNA as a source of transposase. Nucleic Acid Res. 30, 3333-3340.

Klinakis, A. G., Loukeris, T. G., Pavlopoulos, A. and Savakis, C. (2000). Mobility assays confirm the broad host-range activity of the Minos transposable element and validate new transformation tools. Insect Mol. Biol. 9, 269-276.

Kokoza, V., Ahmed, A., Cho, W. L., Jasinskiene, N., James, A. A. and Raikhel, A. (2000). Engineering blood meal-activated systemic immunity in the yellow fever mosquito, Aedes aegypti. Proc. Natl. Acad. Sci. USA 97, 9144-9149.

Kokoza, V., Ahmed, A., Wimmer, E. A. and Raikhel, A. S. (2001). Efficient transformation of the yellow fever mosquito Aedes aegypti using the piggyBac transposable element vector $\mathrm{pBac}[3 \times \mathrm{P} 3-\mathrm{EGFP} \mathrm{afm}]$. Insect Biochem. Mol. Biol. 31, 1137-1143.

Lampe, D. J. and Robertson, H. M. (1996). A purified mariner transposase is sufficient to mediate transposition in vitro. EMBO J. 15, 5470-5479.

Lampe, D. J., Walden, K. K. O., Sherwood, J. M. and Robertson, H. M. (2000). Genetic engineering of insects with mariner transposons. In Transgenic Insects: Methods and Applications (ed. A. M. Handler and A. A. James), pp. 397. Boca Raton: CRC Press.

Lidholm, D., Lohe, A. and Hartl, D. (1993). The transposable element mariner mediates germline transformation in Drosophila melanogaster. Genetics 134, 859-868.

Lobo, N. F., Hua-Van, A., Li, X., Nolen, B. M. and Fraser, M. J. (2002). Germ line transformation of the yellow fever mosquito, Aedes aegypti, mediated by transpositional insertion of a piggyBac vector. Insect Mol. Biol. 11, 133-139.

Lohe, A. R. and Hartl, D. L. (2002). Efficient mobilization of mariner in vivo requires multiple internal sequences. Genetics 160, 519-526.

Lohe, A. R., Lidholm, D. A. and Hartl, D. L. (1995). Genotypic effects, maternal effects and grand-maternal effects of immobilized derivatives of the transposable element mariner. Genetics 140, 183-192.

Lohe, A. R., Timmons, C., Beerman, I., Lozovskaya, E. R. and Hartl, D. L. (2000). Self-inflicted wounds, template-directed gap repair and a recombination hotspot: effects of the mariner transposase. Genetics 154, 647-656.

Lorenzen, M. D., Berghammer, A. J., Brown, S. J., Denell, R. E., Klinger, M. and Beeman, R. W. (in press). piggyBac-mediated germline transformation in the beetle Tribolium castaneum. Insect Mol. Biol.

Loukeris, T. G., Arca, B., Livadaras, I., Dialektaki, G. and Savakis, C. 


\section{D. A. O'Brochta and others}

(1995). Introduction of the transposable element Minos into the germ line of Drosophila melanogaster. Proc. Natl. Acad. Sci. USA 92, 9485-9489.

Lozovsky, E. R., Nurminsky, D., Wimmer, E. A. and Hartl, D. L. (2002). Unexpected stability of mariner transgenes in Drosophila. Genetics 160, 527-535.

Michel, K., Staminova, A., Pinkerton, A. C., Franz, G., Robinson, A. S., Gariou-Papalexiou, A., Zacharaopoulou, A., O'Brochta, D. A. and Atkinson, P. W. (2001). Germline transformation of the medfly, Ceratitis capitata, by Hermes. Insect Mol. Biol. 10, 155-162.

Moreira, L. A., Edwards, M. J., Adhami, F., Jasinskiene, N., James, A. A. and Jacobs-Lorena, M. (2000). Robust gut-specific gene expression in transgenic Aedes aegypti mosquitoes. Proc. Natl. Acad. Sci. USA 97, 1089510898.

Nassif, N., Penney, J., Pal, S., Engels, W. R. and Gloor, G. B. (1994). Efficient copying of nonhomologous sequences from ectopic sites via $P$ element-induced gap repair. Mol. Cell Biol. 14, 1613-1625.

Nolan, T., Bower, M., Brown, A. E., Crisanti, A. and Catteruccia, F. (2002). piggyBac-mediated germline transformation of the malaria mosquito Anopheles stephensi using the red fluorescent protein dsRED as a selectable marker. J. Biol. Chem. 277, 8759-8762.

O'Brochta, D. A., Warren, W. D., Saville, K. J. and Atkinson, P. W. (1996). Hermes, a functional non-drosophilid insect gene vector from Musca domestica. Genetics 142, 907-914.

Perera, O. P., Harrell, R. A. and Handler, A. M. (2002). Germ-line transformation of the South American malaria vector, Anopheles albimanus, with a piggyBac/EGFP transposon vector is routine and highly efficient. Insect Mol. Biol. 11, 291-297.

Pinkerton, A. C., Michel, K., O'Brochta, D. A. and Atkinson, P. W. (2000).
Green fluorescent protein as a genetic marker in transgenic Aedes aegypti. Insect Mol. Biol. 9, 1-10.

Pinkerton, A. C., O'Brochta, D. A. and Atkinson, P. W. (1996). Mobility of $h A T$ transposable elements in the Old World American bollworm, Helicoverpa armigera. Insect Mol. Biol. 5, 223-227.

Robertson, H. M. (1993). The mariner transposable element is widespread in insects. Nature 362, 241-245.

Sarkar, A., Coates, C. C., Whyard, S., Willhoeft, U., Atkinson, P. W. and O'Brochta, D. A. (1997a). The Hermes element from Musca domestica can transpose in four families of cyclorrhaphan flies. Genetica 99, 15-29.

Sarkar, A., Yardley, K., Atkinson, P. W., James, A. A. and O'Brochta, D. A. (1997b). Transposition of the Hermes element in embryos of the vector mosquito, Aedes aegypti. Insect Biochem. Mol. Biol. 27, 359-363.

Tosi, L. R. O. and Beverly, S. M. (2000). Cis and trans factors affecting Mos 1 mariner evolution and transposition in vitro, and its potential for functional genomics. Nucleic Acid Res. 28, 784-790.

Warren, W. D., Atkinson, P. W. and O'Brochta, D. A. (1994). The Hermes transposable element from the housefly, Musca domestica, is a short inverted repeat-type element of the hobo, Ac, and Tam3 (hAT) element family. Genet. Res. Camb. 64, 87-97.

Wilson, R., Orsetti, J., Klocko, A. D., Aluvihare, C., Peckham, E., Atkinson, P. W., Lehane, M. J. and O'Brochta, D. A. (2003). Postintegration behavior of a Mos1 gene vector in Aedes aegypti. Insect Biochem. Mol. Biol. 83, 853-863.

Zhang, H., Shinmyo, Y., Hirose, A., Mito, T., Inoue, Y., Ohuchi, H., Loukeris, T. G., Eggleston, P. and Noji, S. (2002). Extrachromosomal transposition of the transposable element Minos in embryos of the cricket Gryllus bimaculatus. Dev. Growth Differ. 44, 409-417. 